\title{
Identification of temporal factors related to shot performance for indoor Recurve archery
}

\author{
Andrew J. Callaway ${ }^{1}$, Johanna Wiedlack ${ }^{2}$, Mario Heller ${ }^{2}$ \\ ${ }^{1}$ Department of Sport and Physical Activity, Bournemouth University, UK. \\ ${ }^{2}$ Centre for Sport Science and University Sports, University of Vienna, Austria.
}

Corresponding author: Dr. Andrew Callaway

Dept. of Sport and Physical Activity. Bournemouth University. BH12 5BB.

Email: acallaway@bournemouth.ac.uk

Tel: +44(0) 1202961255

Johanna Wiedlack

Centre for Sport Science and University Sports, University of Vienna, Austria

Email: johanna.wiedlack@gmx.at

Dr. Mario Heller

Centre for Sport Science and University Sports, University of Vienna, Austria

Email: mario.heller@ univie.ac.at 


\title{
Identification of temporal factors related to shot performance for indoor Recurve archery
}

\author{
The purpose of this study was to investigate the temporal phases of the archery \\ shot cycle that distinguish the arrows distance from centre, in an attempt to \\ understand critical factors that affect performance. Fifteen archers of varying \\ ability each performed 30 shots at $18 \mathrm{~m}$. Fourteen potential predictor variables \\ were measured for statistical modeling by stepwise multiple linear regression. \\ The results show that pre-shot time (pre-performance routine), release time \\ (post-performance routine), aiming time, speed of the arrow and the \%variation \\ in Clicker to Release time, account for $7.7 \%$ of the variation in predicting shot \\ performance. The results have implications for practice demonstrating factors \\ that coaches should focus on to develop their athletes. Further work on pre-, \\ but more importantly, post-performance routines are needed generally within \\ sport research as they are lacking and have been shown to be important \\ contributing factors in a number of sports.
}

Keywords: Archery; Biomechanics; Performance; Pre-performance routine; Post-performance routine;

\section{Introduction}

The skill of Archery has been practised for millennia, with the current earliest archaeological recordings dating back to 65,000 B.C. (Lombard, 2011). The role of archery has progressed from a necessity for survival and hunting (Lombard, 2011) through warfare, a recreational past time, to become an Olympic sport. High performance in Olympic archery can be defined as the ability to shoot an arrow at a given target with high accuracy (Edelmann-Nusser, Heller, Hofmann, \& Ganter, 2006; Ertan, Kentel, Tümer, \& Korkusuz, 2003; Ertan, Knicker, Soylu, \& Strüder, 2011; Leroyer, Van Hoecke, \& Helal, 1993). To measure shot accuracy, the straight- 
line distance of the arrow to the target centre can be used which can develop the precision of analysis over the use of score (Callaway \& Broomfield, 2012).

Despite the long associated history of archery, the majority of our present understanding and its mechanics have been derived from empirical observations made by coaches and athletes

To fill this dearth, there has been a steady and concerted effort to develop the scientific cognizance of various technique aspects of archery, which has generally been demonstrated through electromyographic (EMG) investigations.

Leroyer et al. (1993) using EMG discovered a relationship between ability level and the regularity of the back muscle tremor during the final push-pull phase. Edelmann-Nusser et al. (2006) confirmed these findings whilst investigating aiming trajectories, concluding that 'smooth' final push-pulls in archery were needed in order to achieve high scores.

The practical doctrine observed by many coaches suggests that there is a forearm relaxation only during release. Early discoveries suggest that the release should be balanced to maintain good scores (Nishizono, Shibayama, Izuta, \& Saito, 1987). Martin, Siler, and Hoffman (1990) investigated this further using the EMG characteristics of a variety of archers' releases of the bow string. They concluded that other factors than the bowstring release mechanism discriminate the performances of skilled archers. However, Ertan et al. (2003) advanced this understanding by identifying a contraction-relaxation stratagem used during the release phase of the shot in the forearm extensors. These results demonstrate a contradiction to anecdotal evidence.

Alongside EMG investigations, the beginnings of inquiries into the effect of the phases of the shot have taken place. Lin and Hwang (2005) investigated the 
aiming duration in relation to the score of the arrow concluding that the archery performance, especially those in low scores can be improved by shortening the aiming time. The phases have also been investigated from a psycho-physiological point of view. There is evidence that during the aiming phase, neural activity differentiates performance by skill level. Kim et al. (2014) investigated brain activity in elite, expert and novice archers during a simulated archery aiming task by means of functional magnetic resonance imaging (fMRI). They found more localized neural activity for elite and expert archers when compared to novices, permitting greater efficiency of the complex processes by these regions.

Another phase often used is the Clicker-Release time. This is a metal device on the bow, which 'clicks' when the arrow reaches a set length at the end of the aiming phase. Heller (2012) observed a relationship between the score of the arrow and the coefficient of variation of Clicker-Release time. This suggests that the repeatability or reproducibility of the motor program with respect to the timing process would be a performance indicator reflecting the ability of the motor system to carry out the specific task. To record the shooting results to a greater level of detail than just score, the arrow location was further subdivided into a $1 / 10^{\text {th }}$ of the distance within the scoring zone (e.g. 9.3), setting the absolute centre of the target to 11 points. All of the previous works have measured various factors during various phases of the shot cycle. However, there appears to be no consensus between previous works (Table 1). 


\begin{tabular}{ll}
\hline Authors & Phases Used \\
\hline Nishizono et al. (1987) & $\begin{array}{l}\text { Bow hold, drawing, full draw, aiming, release, follow } \\
\text { through }\end{array}$ \\
Leroyer et al. (1993) & Stance, arming, sighting \\
Takai, Kubo, and Araki (2012) & $\begin{array}{l}\text { Stance, set, nocking, setup, drawing, full draw, release, } \\
\text { follow through }\end{array}$ \\
\hline
\end{tabular}

Table 1: Previous identification of the phases of the archery shot cycle

Hughes and Bartlett (2002) state that clear definitions of key performance indicators are needed to allow repeatability. Williams (2013) found that there is a need for consensus of definitions as it is generally lacking from research. As demonstrated here, there is convolution in the definition of terms or they are entirely lacking in most research presented in this area. There is no delineation between a phase within the shot cycle and a trigger event/action for the transition between phases, or whether it is in fact a biomechanical classification or modality. This can be demonstrated with the stance; it has been used frequently for a phase but is a biomechanical term for how the archer stands. This can be classified as square, open or closed (Axford, 1995) or oblique (Stone, 2007) or degrees of variation therein. This demonstrates that there are issues in the identification of the phases of the shot and there needs to be clear, repeatable definitions.

Despite the work evidenced here, there has been little research examining the techniques used in shooting (Martin et al., 1990). Little is known as to what contributes to successful, accurate performances. Experiential evidence has suggested that the temporal phases of the shot are important to performance (Haywood \& Lewis, 
2013) but most focus within research has been given to the aiming and release phase as contributing factors to performance as previously demonstrated. The phases of the shot offer a readily observable method of analysis that could be used to assess an athlete's performance, track progression and change. There has been limited work to investigate whether the phases of the shot contribute significantly to shot performance. The aim of this paper is to determine whether the temporal phases of the shot cycle relate to the outcome of the shot. This would allow coaches and researchers to focus their respective attentions to improve and develop performance from observable measures.

\section{Method}

\section{Participants}

With institutional ethical approval, and informed consent, fifteen (15) archers participated in the study (3 Female, 12 Male, Age: $41.0( \pm 11.9)$, FITA Score: 1182.6 ( \pm 78.2$)$ out of 1440, Years of Experience: $19.6( \pm 11.2))$. The archers ranged in ability, from university, through county to national and international level.

\section{Phases of the shot}

Previous studies in Archery have defined the phases of the shot in a variety of ways (Table 1). Based on the previous literature this work has defined the phases of the shot cycle, from when the archer is already standing on the shooting line, as: preshot routine, set-up routine, drawing, aiming, clicker-release time, and follow through. The events that trigger these can be seen in Figure 1Error! Reference source not found. 


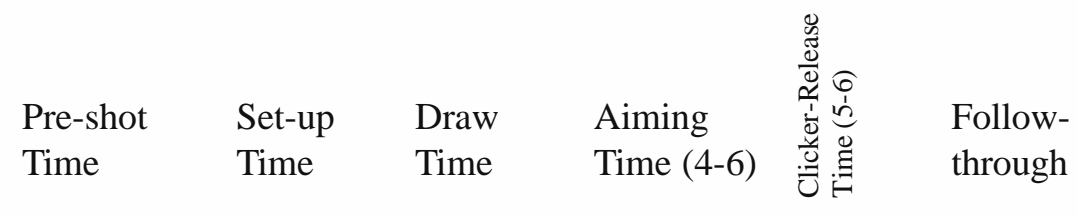

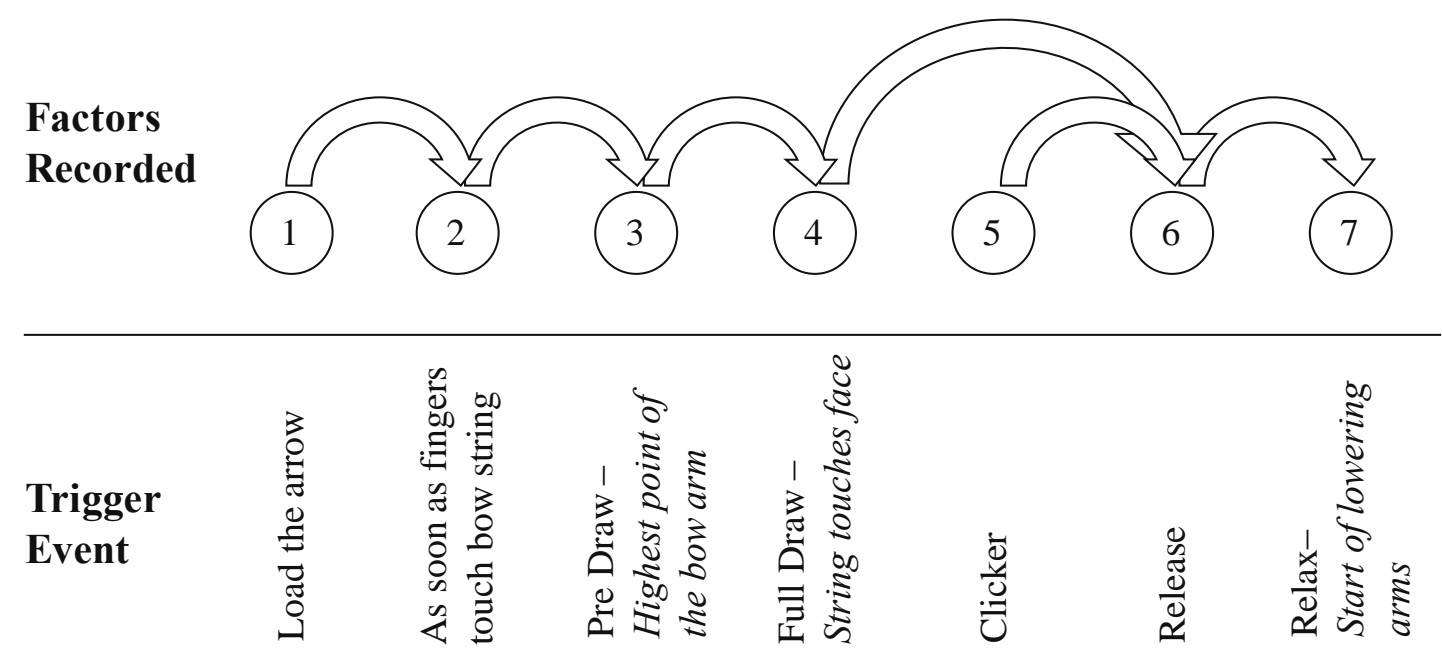

Figure 1. Phases and triggers for the Recurve shot cycle

With reference to Figure 2Error! Reference source not found., the pre-shot phase starts when the archer touches the arrow (1) in their quiver until the archer fingers are placed on the string (2). The set-up phase starts from (2) and finishes when the bow arm is raised to the highest point before the draw hand is moved backwards (3). The draw phase finishes when the string touches the face of the archer (4). The aiming phase finishes when the string moves forwards from the fingers (the release) (6). The Clicker-Release time is from when the clicker sounds (5) until the string moves forwards from the fingers (6). The follow through time stops when the archer first moves the either arm downwards from their finishing position, a relaxation from their finishing pose (7). 


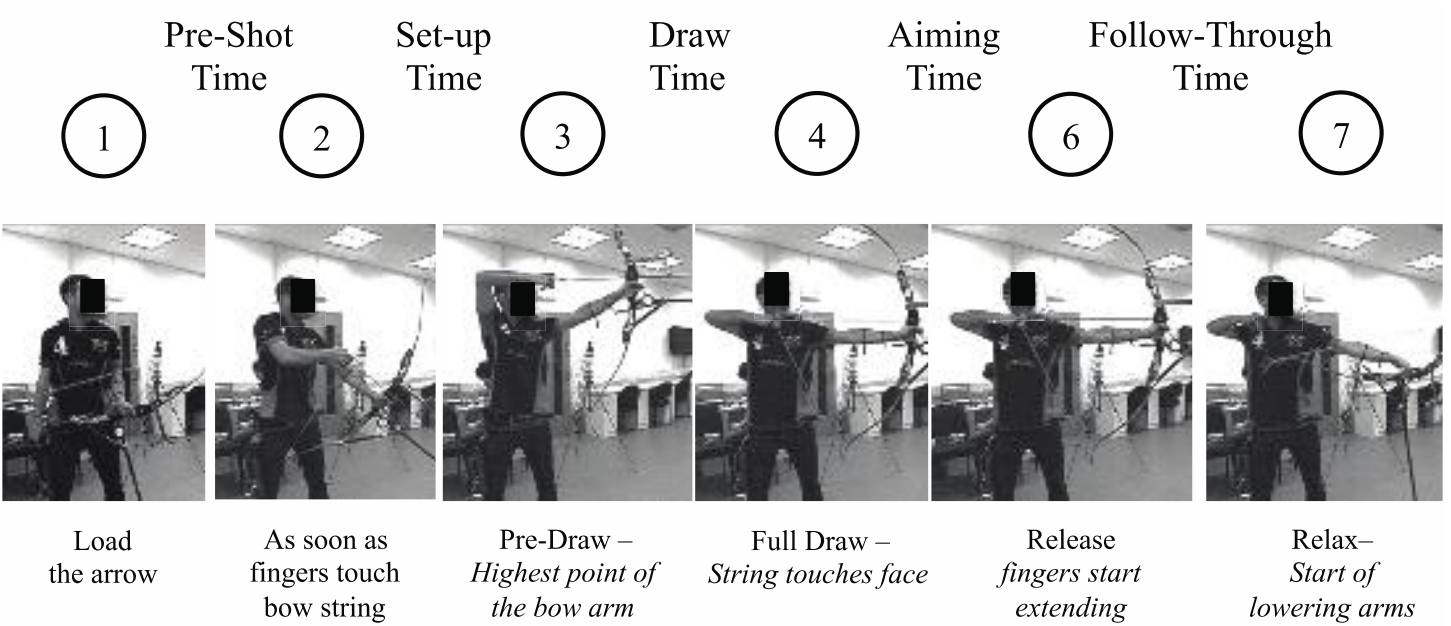

Figure 2. Visual representation of the phases

The Clicker-Release time of the archer has been recorded acoustically by Heller (2012). The raw audio signal allows the detection of the following critical time events: a) the time of the clicker's fall $(5), b)$ the release of the shot (6) (Figure 3). The Clicker-Release time is calculated as (6)-(5).

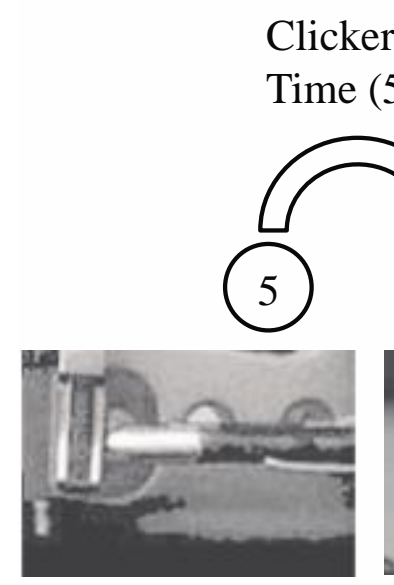

Clicker a few hundredths of a second
after release acoustic signal

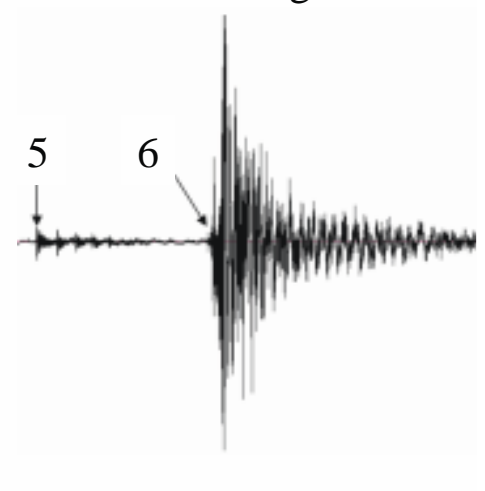

(a)

(b)

Figure 3. Clicker to release time 


\section{Procedure}

Each archer, after their own warm up and using their own archery equipment, performed 30 shots (10 ends of 3 arrows, with no time limits) at a 3 spot vertical $40 \mathrm{~cm}$ target at a distance of $18 \mathrm{~m}$ in laboratory controlled conditions $(n=450)$.

To manually record the temporal phases of the shot, manual high speed video analysis was used. A Casio Exilim FH-25 recording at $210 \mathrm{~Hz}$ was placed perpendicular to the archer's shooting direction, zoomed to ensure the whole archer was in shot when the bow was raised toward the target. The videos were analysed frame-by-frame by an expert to extract the temporal factors for each shot phase using the definitions provided (Figure 1 and Figure 2).

The Clicker-Release time was recorded using a microphone (AKG C $480 \mathrm{~B}$ comb-ULS/61) directly connected by a microphone cable (CME 220, CORDIAL) with XLR connectors to an external FireWire Audio Interface (Focusrite Saffire LE). The microphone was placed at the archer's shoulder height, $0.5 \mathrm{~m}$ behind the archer, in the direction of the bow. The audio signal is digitized at $24 \mathrm{Bit} / 96 \mathrm{kHz}$, and was transmitted to a laptop using a sound editor and recording software (Audacity). Speed of the arrow was calculated as the time from the Clicker-Release time to the sound of the arrow impact on the target, also determined from the audio signal (Heller, 2012).

\section{Analysis}

Outliers for each phase within-archer (Pre, setup, draw, aiming, Clicker-Release time, follow-through, total, speed) were removed by calculating the residuals and using a threshold $\langle \pm 3.5>$, following sample size based recommendations by Hopkins, Marshall, Batterham, and Hanin (2009). These cleaned data (Pre, setup, draw, aiming, 
Clicker-Release time, follow-through, speed) were then used calculate the withinarcher \%variation of each shot relative to the archers mean (of 30 shots).

Outcome of the shot was assessed using individual arrow locations, rather than the score of the shot. These were recorded as a coordinate location relative to the target centre $(0,0)$, which has been previously validated (Callaway \& Broomfield, 2012). From this, each arrow's straight-line distance to the target centre was calculated using Pythagoras theorem. A smaller straight-line distance indicates a better shot performance. This is principally the same as the process used by Heller (2012) sub-dividing the score of the arrow.

A stepwise multiple linear regression analysis was conducted to identify factors influencing the outcome of the shot (straight-line distance to centre). The Breusch-Pagan test was used to test heteroscedasticity of the best-fit model residuals. The level of significance was set to $\alpha=.05$. 


\section{Results}

Descriptive statistics of the 14 variables tested are shown in Table 2. Multiple linear regression was performed on these variables, five models were created (Table ).

\begin{tabular}{lcrrrr}
\hline Factor & $\mathrm{N}$ & Minimum & Maximum & \multicolumn{1}{c}{ Mean $( \pm \mathrm{SD})$} \\
\hline Speed (KPH) & 445 & 190.100 & 246.300 & $223.436(11.90)$ \\
Pre-Shot Time (s) & 414 & 5.862 & 29.662 & $13.593 \quad(4.81)$ \\
Setup Time (s) & 448 & 1.138 & 4.409 & $2.174 \quad(0.68)$ \\
Draw Time (s) & 448 & 0.920 & 5.086 & 2.508 & $(0.77)$ \\
Aiming Time (s) & 449 & 0.419 & 10.138 & $3.490 \quad(1.73)$ \\
CRT (ms) & 448 & 99.000 & 237.600 & $173.128(23.73)$ \\
Follow-Through Time (s) & 446 & 0.095 & 2.362 & $1.344 \quad(0.40)$ \\
& & & & & \\
Speed \%Var & 445 & 0.005 & 4.995 & $0.470 \quad(0.49)$ \\
PreShot \%Var & 414 & 0.059 & 86.850 & $14.301(12.53)$ \\
Setup \%Var & 448 & 0.040 & 53.671 & $7.117 \quad(7.70)$ \\
Draw \%Var & 448 & 0.010 & 41.283 & $8.290(6.85)$ \\
Aiming \%Var & 449 & 0.116 & 102.168 & $24.158(19.87)$ \\
CRT \%Var & 448 & 0.004 & 44.032 & $6.079(6.36)$ \\
Follow-Through \%Var & 446 & 0.024 & 104.744 & $13.730(16.76)$ \\
& & & & & \\
\hline
\end{tabular}

Table 2. Descriptive statistics 


$\begin{array}{lll}1 & \text { CRT \%Var } & 0.018 \\ 2 & \begin{array}{l}\text { CRT \%Var } \\ \text { Speed }\end{array} & 0.037 \\ 3 & \begin{array}{l}\text { CRT \%Var } \\ \text { Speed }\end{array} \\ & \text { Pre-Shot } \\ 4 & \begin{array}{l}\text { CRT \%Var } \\ \text { Speed } \\ \text { Pre-Shot } \\ \text { Aiming }\end{array} \\ \text { CRT \%Var } & 0.053 \\ \text { Speed } & \\ \text { Pre-Shot } & \\ \text { Aiming } & \text { Follow-Through } & \\ & \end{array}$

Table 3. Multiple linear regression models to predict straight-line distance of the arrow to the target centre

The best predictors of straight-line distance (SLD) of the arrow to the target centre are demonstrated by model five. The stepwise multiple linear regression analyses showed that Clicker-Release time \%Variation $(\beta=.130, p=.009)$, Speed $(\beta$ $=-.148, p=.004)$, Pre-shot time $(\beta=.165, p=.002)$, Aiming time $(\beta=-.118, p=$ $.02)$ and Follow-through time $(\beta=0.98, p=.045)$ created the best predictors. The Breusch-Pagan test was used on this model to check for heteroscedasticity in the residuals. This demonstrated that the residual for this model were homoscedastic in nature $(p=.586)$. 
The overall model fit (Clicker-Release time \%Variation, Speed, Pre-Shot, Aiming time and Follow-Through) was significant, Adjusted $R^{2}=.077(F 5,395)=$ $6.637(p<.001)$.

\section{Discussion}

Previous work looking at EMG has been useful to the sport by allowing scientists to better understand some of the motor control patterns involved in the shot sequence. However, we know that transfer of science into practical terms can often take a while, or be quite poor (Bishop, 2008; Mason \& Portus, 2005). The limitation of EMG based work, currently, is that coaches will not have access to the necessary equipment and knowledge to use it.

The primary purpose of this study was to investigate whether the temporal phases of the shot cycle relate to the outcome of the shot. Using temporal phases of the shot offers coaches and scientists a straightforward method of data collection and analysis to determine the effect of interventions. The results show that a model consisting of Clicker-Release time \%Variation, Speed, Pre-Shot, Aiming, and FollowThrough time can account for $7.7 \%$ of the variability within the arrows location from the centre of the target. This is an important finding as we know that any small percentage improvement in athletic performance is a worthwhile goal (Hopkins, 2005).

Heller (2012) identified that the coefficient of variation of Clicker-Release time was a key factor in the archery shot cycle relative to the average score of the shot using a homogeneous subset of the population (Atkinson \& Nevill, 2001). The population was constructed of highly skilled archers all from the same club, with the 
same trainer, the same teaching guide and therefore similar motor skill learning for a number of years. Despite this homogeneous subset, the results of Clicker-Release time \%variation are transferable to the lager population in this work. The population here included a range from university level through to experienced national and international level, from a variety of clubs and with different coaches and years of experience, demonstrating a wider sample of the available population.

The results also show that speed is an important aspect to consider, contradicting the old adage "a slow 10 is better than a fast miss". If this study were to have focused on highly-skilled archers, then speed may not have been a factor as the draw weight (poundage) of the bows will have been very similar results in a smaller range of speed and likely not been a contributing factor. This could suggest that with a wider population range that coaches of lower level archers do need to consider speed as an important factor. However, there needs to be care with the practical implementation of this. It can be easy for coaches to consider increasing speed of the arrow through increasing bow weight (poundage). Archery is a skill that involves repetitive precise movements. Increasing the poundage before the physical development of the athlete could encumber the ability to develop this precision motor control. Furthermore, there needs to be consideration for the potential injury risk where archers will not be able to manage the poundage, which could lead to shoulder injuries, one of the most common injuries in archers (Mann \& Littke, 1989).

Instead, speed can be generated in other ways with adjustments to the bow including tiller, bracing height and strands in the string; adjusting arrow construction via drag and the smoothness of the pile (point) of the arrow (Miyazaki et al., 2013); and also to the archers' release technique. The relationship between score and the lateral deflection of the archers' release has been demonstrated by Horsak and Heller 
(2011). Logically, if the string has further to move before the arrow leaves the string then there will be a decrease in the speed of the arrow leading to a horizontal deflection as well as the lateral deflection observed by Horsak and Heller (2011). Therefore, coaches should consider the biomechanical elements of the release before increasing the poundage of the bow.

Aiming time has been identified by Lin and Hwang (2005). They used 6 archers shooting at $30 \mathrm{~m}$ all from the same University team, similar in nature to the demographic used by Heller (2012). Assessing the archers individually they identified some relationships between the radial distance of the arrow (representing score) and the aiming time, with non-significant correlations ranging from $r=0.056$ to 0.249 .

Keast and Elliott (1990) also investigated aiming time and found a relationship. The shots' aiming time fell in the range of $2.12-5.82 \mathrm{~s}$, and as the aiming time of the shot increased, the score of the arrow decreased.

The results from this study show a negative relationship between shot performance and the aiming time $(r=-.117 ; p=.013, n=449)$, so as the archer holds aims for longer, the arrow goes nearer the middle.

These results of the current work therefore seem counterintuitive when considering that elongating the aiming time will affect the release of the arrow due to muscle fatigue, and in turn affect the vertical deviation of the arrow (Lin \& Hwang, 2005). An explanation for this could be the distance shot by the archers. Coaching doctrine suggests that archers tend to "over-aim" at closer distances, for example, during the indoor season where they only shoot $18 \mathrm{~m}$. This can cause archers to lose the rhythm of their shot, and would explain the negative correlation in the results. This does demonstrate a methodological consideration for future work with regards to the distances the archer shoots may have a different outcome on the regression model 
shown here and should be examined further. Therefore, this work should only be applied to indoor shooting.

Two of the shot phases (Pre-Shot and Follow-Through) can be viewed as part of the feedback loop, allowing time to perform pre-performance and postperformance routines, respectively. Pre-performance routines can aid in retarding debilitating thoughts, and has the effect of preserving or increasing performance by offering a rhythmical guide to the performance (MacPherson, Collins, \& Obhi, 2009). Pre-performance routines have been shown to be important in enhancing performance in closed skilled sports (Cotterill, 2011; Hazell, Cotterill, \& Hill, 2014; Lonsdale \& Tam, 2008).

Follow-Through time could be seen as an opportunity to perform a postperformance routine. Hill, Hanton, Matthews, and Fleming (2010) were the first to identify the benefits of post-performance routines. Hill et al. (2010) interviewed six elite golfers that suffered from choking under pressure, and five elite golfers that excelled under pressure. Golfers that excelled under pressure performed consistent post-performance routines after each shot; those who choked rarely or intermittently completed a post-performance routine. However, post-performance routines have not received a great deal of attention in the current literature and more work is needed to understand the internal workings of them (Mesagno, Hill, \& Larkin, 2015).

The regression model produced here demonstrates phases that coaches can use to allow them to develop external focused coaching points using timing aids. External focused coaching points have been shown to aid the learning process (Wulf, 2007; Wulf, Chiviacowsky, Schiller, \& Ávila, 2010; Wulf, Lauterbach, \& Toole, 1999; Wulf, Mcconnel, Gärtner, \& Schwarz, 2002). In addition to this, the PPR and POST demonstrate the control of rhythm within the shots. This should lead coaches to focus 
on the rhythm-focused interventions rather than component-specific interventions which would be a significant asset to the athlete (MacPherson et al., 2009).

\section{Conclusion}

Variation in Clicker-Release time, speed of the arrow, pre-shot and post shot time, in addition to the aiming time all contribute to predicting the distance of the arrow to the target centre. This demonstrates areas for coaches and scientists to focus their future efforts in developing archers' performances.

\section{References}

Atkinson, G., \& Nevill, A. M. (2001). Selected issues in the design and analysis of sport performance research. Journal of Sports Sciences, 19(10), 811-827

Axford, R. (1995). Archery Anatomy - An Introduction to Techniques Improved Performance. London: Souvenir Press Limited.

Bishop, D. (2008). An applied research model for the sport sciences. Sports Medicine, $38(3), 253-263$

Callaway, A. J., \& Broomfield, S. A. (2012). Inter-Rater Reliability and Criterion Validity of Scatter Diagrams as an Input Method for Marksmanship Analys is: Computerised Notational Analysis for Archery. International Journal of Performance Analysis in Sport, 12(2), 291-310

Cotterill, S. T. (2011). Experiences of developing pre-performance routines with elite cricket players. Journal of Sport Psychology in Action, 2(2), 81-91

Edelmann-Nusser, J., Heller, M., Hofmann, M., \& Ganter, N. (2006). On-target trajectories and the final pull in archery. European Journal of Sport Science, 6(4), 213-222. doi: 10.1080/17461390601012579

Ertan, H., Kentel, B., Tümer, S. T., \& Korkusuz, F. (2003). Activation patterns in forearm muscles during archery shooting. Human Movement Science, 22(1), 37-45. doi: 10.1016/s0167-9457(02)00176-8

Ertan, H., Knicker, A., Soylu, R., \& Strüder, H. (2011). Individual variation of bowstring release in high level archery: a comparative case study. Human Movement, 12(3), 273-276. doi: 10.2478/v10038-011-0030-x

Haywood, K. M., \& Lewis, C. F. (2013). Archery: Steps to Success (Fourth ed.): Human Kinetics.

Hazell, J., Cotterill, S. T., \& Hill, D. M. (2014). An exploration of pre-performance routines, self-efficacy, anxiety and performance in semi-professional soccer.

European Journal of Sport Science, 14(6), 603-610. doi: 10.1080/17461391.2014.888484

Heller, M. (2012). Evaluation of arrow release in highly skilled archers using an acoustic measurement system. Procedia Engineering, 34, 532-537. doi: 10.1016/j.proeng.2012.04.091 
Hill, D. M., Hanton, S., Matthews, N., \& Fleming, S. (2010). A qualitative exploration of choking in elite golf. Journal of Clinical Sport Psychology, 4(3), 221-240

Hopkins, W. G. (2005). Competitive performance of elite track-and-field athletes: variability and smallest worthwhile enhancements. Sportscience, 9, 17-20

Hopkins, W. G., Marshall, S., Batterham, A., \& Hanin, J. (2009). Progressive statistics for studies in sports medicine and exercise science. Medicine \& Science in Sports \& Exercise, 41(1), 3-12

Horsak, B., \& Heller, M. (2011). A three-dimensional analysis of finger and bow string movements during the release in archery. Journal of Applied Biomechanics, 27(2), 151-160

Hughes, M. D., \& Bartlett, R. M. (2002). The use of performance indicators in performance analysis. Journal of Sports Sciences, 20(10), 739-754. doi: $10.1080 / 026404102320675602$

Keast, D., \& Elliott, B. (1990). Fine body movements and the cardiac cycle in archery. Journal of Sports Sciences, 8(3), 203-213. doi: 10.1080/02640419008732146

Kim, W., Chang, Y., Kim, J., Seo, J., Ryu, K., Lee, E., .. . Janelle, C. M. (2014). An fMRI study of differences in brain activity among elite, expert, and novice archers at the moment of optimal aiming. Cognitive and Behavioral Neurology, 27(4), 173-182

Leroyer, P., Van Hoecke, J., \& Helal, J. N. (1993). Biomechanical study of the final push-pull in archery. Journal of Sports Sciences, 11(1), 63-69. doi: 10.1080/02640419308729965

Lin, K., \& Hwang, C. (2005). Analyses of the relationship between the aiming time and the shot points in archery. Journal of Physical Education in Higher Education, 7(4), 161-173

Lombard, M. (2011). Quartz-tipped arrows older than 60 ka: further use-trace evidence from Sibudu, KwaZulu-Natal, South Africa. Journal of Archaeological Science, 38(8), 1918-1930. doi: 10.1016/j.jas.2011.04.0 01

Lonsdale, C., \& Tam, J. T. (2008). On the temporal and behavioural consistency of pre-performance routines: An intra-individual analysis of elite basketball players' free throw shooting accuracy. Journal of Sports Sciences, 26(3), 259266

MacPherson, A. C., Collins, D., \& Obhi, S. S. (2009). The importance of temporal structure and rhythm for the optimum performance of motor skills: a new focus for practitioners of sport psychology. Journal of Applied Sport Psychology, 21(S1), S48-S61. doi: 10.1080/10413200802595930

Mann, D., \& Littke, N. (1989). Shoulder injuries in archery. Canadian Journal of Sport Sciences, 14(2), 85-92

Martin, P.E., Siler, W. L., \& Hoffman, D. (1990). Electromyographic analysis of bow string release in highly skilled archers. Journal of Sports Sciences, 8(3), 215221. doi: 10.1080/02640419008732147

Mason, B. R., \& Portus, M. (2005). Essay: Biomechanical support in sport. Lancet, 366 Suppl 1, S25-S26

Mesagno, C., Hill, D. M., \& Larkin, P. (2015). Examining the accuracy and in-game performance effects between pre-and post-performance routines: A mixed methods study. Psychology of Sport and Exercise, 19, 85-94. doi: 10.1016/j.psychsport.2015.03.005 
Miyazaki, T., Mukaiyama, K., Komori, Y., Okawa, K., Taguchi, S., \& Sugiura, H. (2013). Aerodynamic properties of an archery arrow. Sports Engineering, $16(1), 43-54$

Nishizono, H., Shibayama, H., Izuta, T., \& Saito, K. (1987). Analysis of archery shooting techniques by means of electromyography Proceedings of the 5th International Symposium of Biomechanics in Sport (pp. 364-372). Athens, Greece.

Stone, R. T. (2007). The Biomechanical and Physiological link between Archery Techniques and Performance Proceedings of the Human Factors and Ergonomics Society Annual Meeting (Vol. 51, pp. 1227-1231): SAGE Publications.

Takai, H., Kubo, Y., \& Araki, M. (2012). Characteristics of Shooting Time of The World's Top Level Male Archery Athletes. NSSU Journal of Sport Sciences, $1,8-12$

Williams, J. J. (2013). Operational definitions in performance Analysis and the need for consensus. International Journal of Performance Analysis in Sport, 12, 5263

Wulf, G. (2007). Attentional focus and motor learning: A review of 10 years of research. E-journal Bewegung und Training, 1(2-3), 1-11

Wulf, G., Chiviacowsky, S., Schiller, E., \& Ávila, L. T. G. (2010). Frequent external focus feedback enhances motor learning. Movement Science and Sport Psychology, 1, 190

Wulf, G., Lauterbach, B., \& Toole, T. (1999). The learning advantages of an external focus of attention in golf. Research Quarterly for Exercise and Sport, 70(2), $120-126$

Wulf, G., Mcconnel, N., Gärtner, M., \& Schwarz, A. (2002). Enhancing the learning of sport skills through external-focus feedback. Journal of Motor Behavior, 34(2), 171-182 